\title{
Some Infinite Sums Involving Zeros of $J_{0}(x)$
}

\section{Leo F. Epstein *}

(April 15, 1963)

The limiting form for small $\phi$ values of the sums

$$
\begin{aligned}
F_{r}(\phi) & \equiv \sum_{n=1}^{\infty}\left(1 / \beta_{n}\right)^{2 r} \exp \left(-\beta_{n}{ }^{2} \phi\right) \\
G_{r}(\phi) & \equiv \sum_{n=1}^{\infty} \frac{\exp \left(-\beta_{n}^{2} \phi\right)}{\beta_{n}^{2 r-1} J_{1}\left(\beta_{n}\right)} \\
M_{r}(\phi) & \equiv \sum_{n=1}^{\infty}\left(1 / \beta_{n}\right)^{2 r-1} \operatorname{erfc}\left(\beta_{n} \sqrt{\phi}\right)
\end{aligned}
$$

and

$$
N_{r}(\phi) \equiv \sum_{n=1}^{\infty} \frac{\operatorname{erfc}\left(\beta_{n} \sqrt{\phi}\right)}{\beta_{n}^{2 r} J_{1}\left(\beta_{n}\right)}
$$

where $r$ is an integer and $\beta_{n}$ is the $n$th zero of the Bessel function $J_{o}(x)$, have been obtained. Related functions which arise in these problems are also considered.

\section{Introduction}

In the study of a physical problem involving diffusion processes in cylindrical geometry, a number of infinite sums have been encountered of the form

$$
\begin{gathered}
F_{r}(\phi) \equiv \sum_{n=1}^{\infty}\left(1 / \beta_{n}\right)^{2 r} \exp \left(-\beta_{n}{ }^{2} \phi\right) \\
G_{r}(\phi) \equiv \sum_{n=1}^{\infty} \frac{\exp \left(-\beta_{n}{ }^{2} \phi\right)}{\beta_{n}{ }^{2 r-1} J_{1}\left(\beta_{n}\right)}
\end{gathered}
$$

where $\beta_{n}$ is the $n$th zero of the Bessel function $J_{0}(x)$. For these functions, the limiting behavior for small $\phi$ values is required. The technique of evaluation involves contour integration, the use of Laplace transforms, and several other methods of some interest, and the results given below are, except as noted, not known to be recorded elsewhere. In the cylindrical geometry problem in which these functions, and the related $M_{r}(\phi)$ and $N_{r}(\phi)$ introduced later, were found to occur, the $\phi \rightarrow 0$ solutions examined correspond to the short time behavior of the system (see eq (4-6) below). It has been found that these quantities are frequently encountered in studies of diffusion and related phenomena, heat conduction for example.

The asymptotic expression $[1, \text { p. } 505]^{1}$ for $\beta_{n}$ is

$$
\operatorname{Lim}_{n \rightarrow \infty} \beta_{n} \equiv \beta_{n}^{*} \approx \sum_{j=0}^{j_{\min }-1} \alpha_{j}\left[\pi\left(n-\frac{1}{4}\right)\right]^{1-2 j}
$$
\footnotetext{
${ }^{*}$ Vallecitos Atomic Laboratory, General Electric Company, Atomic Power Department, Pleasanton, Calif. This paper was first issued as a G. E. internal mem-
orandum, GEAP-3853, dated July 1 , 1961 .

'Figures in brackets indicate the literature references at the end of this paper.
} 
where the first few coefficients of the series are

$$
\begin{aligned}
& \alpha_{0}=+1 \\
& \alpha_{1}=+1 / 8 \\
& \alpha_{2}=-31 / 384 \\
& \alpha_{3}=+3779 / 15360
\end{aligned}
$$

$j_{\min }$ is the value of the index for which the summand is a minimum, so that the series is terminated at the term just before the smallest [2, p. 140].

From (2), it is immediately apparent that the series in (1A) and (1B) converge, and that $F_{r}(\phi)$ and $G_{r}(\phi)$ exist for all positive real values of $r$ and $\phi$.

\section{Evaluation of $F_{1}(\phi)$}

To evaluate $F_{1}(\phi)$, consider the function

$$
f(\phi)=\int_{C} \frac{e^{-z^{2} \phi}}{z^{2}} \frac{J_{1}(z)}{J_{0}(z)} d z
$$

integrated about a contour ${ }^{2}$ consisting of two lines parallel to the real axis and at an arbitrary distance, plus and minus, from it large enough to include $z= \pm i p^{1 / 2}$. Applying Cauchy's theorem $[3]$,

$$
f(\phi)=+i \pi\left(\operatorname{Lim}_{z \rightarrow 0}\left[z \frac{e^{-z^{2} \phi}}{z^{2}} \cdot \frac{J_{1}(z)}{J_{0}(z)}\right]+\sum_{n=1}^{\infty} \operatorname{Lim}_{z \rightarrow \beta_{n}}\left[2\left(z-\beta_{n}\right) \frac{e^{-z^{2} \phi}}{z^{2}} \cdot \frac{J_{1}(z)}{J_{0}(z)}\right]\right)
$$

where the two limits are the residues of the poles of the integral at zero and $\beta_{n}$ respectively. The first term here is $i \pi / 2$ as can be found from the leading terms in the series for $J_{n}(z)$. By expanding the summand in a Taylor's series about $z=\beta_{n}$, the second term can also be evaluated, and it is found that

$$
(2 / i \pi) f(\phi)=1-4 \sum_{n=1}^{\infty}\left(1 / \beta_{n}^{2}\right) \exp \left(-\beta_{n}^{2} \phi\right)=1-4 F_{1}(\phi) .
$$

Taking the Laplace transform, defined by [4]

of both sides of $(2-3)$

$$
L_{p}[g(\phi)] \equiv \int_{0}^{\infty} g(\phi) \exp (-p \phi) d \phi
$$

$$
L_{p}\left[1-4 F_{1}(\phi)\right]=(2 / i \pi) \int_{C} \frac{1}{z^{2}\left(p+z^{2}\right)} \frac{J_{1}(z)}{J_{0}(z)} d z .
$$

The integral here has a pole at $z=i p^{1 / 2}$ and the residue at this pole is readily found. The value of the integral, then, is $2 \pi i$ times this residue [3], and

where [5, p. 372]

$$
L_{p}\left[1-4 F_{1}(\phi)\right]=+2 p^{-3 / 2} \frac{I_{1}(\sqrt{p})}{I_{o}(\sqrt{p})}
$$

$$
I_{n}(z) \equiv i^{-n} J_{n}(i z)
$$

Using the asymptotic form [1, p. 203] for $I_{n}(z)$ for large $z$

$$
I_{n}(z) \approx \frac{e^{z}{ }^{m_{\text {min }}}{ }^{-1}}{(2 \pi z)^{1 / 2}} \sum_{m=0}(-2 z)^{-m} \frac{\Gamma\left(n+m+\frac{1}{2}\right)}{\Gamma\left(n-m+\frac{1}{2}\right)} \frac{1}{m !}
$$

${ }^{2}$ The technique used here was suggested by Dr. G. M. Roe of the General Electric Research Laboratory, Schenectady, N.Y. (private communication June 13, 1955). 
it follows that as $p \rightarrow \infty$

$$
L_{p}\left[1-4 F_{1}(\phi)\right] \approx+2 p^{-3 / 2}-p^{-2}-(1 / 4) p^{-5 / 2}-(1 / 4) p^{-3}+\ldots
$$

This form suggests letting

$$
1-4 F_{1}(\phi)=\sum_{n=1}^{\infty} a_{n} \phi^{n / 2}
$$

and taking the Laplace transform of this expression [4, p. 137]

$$
\begin{gathered}
L_{p}\left[1-4 F_{1}(\phi)\right] \\
=(1 / 2) ! a_{1} p^{-3 / 2}+(1) ! a_{2} p^{-2}+(3 / 2) ! a_{3} p^{-5 / 2}+(2) ! a_{4} p^{-3}+\ldots
\end{gathered}
$$

Comparing the coefficients of like powers of $p$ in (7) and (9) yields the values of the $a$ 's and for small $\phi$

$$
\begin{gathered}
F_{1}(\phi) \\
\approx+(1 / 4)-(1 / \sqrt{\pi}) \phi^{1 / 2}+(1 / 4) \phi+(1 / 12)(1 / \sqrt{\pi}) \phi^{3 / 2}+(1 / 32) \phi^{2}+\ldots
\end{gathered}
$$

Note that, from this equation, using the notation of Watson [1, p. 502]

$$
\sigma_{0}^{(1)} \equiv \sum_{n=1}^{\infty} 1 / \beta_{n}^{2}=F_{1}(0)=1 / 4
$$

a well-known result.

Differentiation of (2-10) with respect to $\phi$ yields, for small $\phi$

$$
\begin{gathered}
F_{0}(\phi) \\
\approx(1 / 2)(1 / \sqrt{\pi}) \phi^{-1 / 2}-(1 / 4)-(1 / 8)(1 / \sqrt{\pi}) \phi^{+1 / 2}-(1 / 16) \phi+\ldots .
\end{gathered}
$$

It is of interest to compare (12) with the similar expression [6, par. 6.491, p. 129] in which $\beta_{n}$ is replaced by $n$

$$
\sum_{n=1}^{\infty} \exp \left(-n^{2} \phi\right)=(\sqrt{\pi} / 2) \phi^{-1 / 2}-(1 / 2)+\sqrt{\pi} \phi^{-1 / 2} \sum_{n=1}^{\infty} \exp \left(-n^{2} \pi^{2} / \phi\right) .
$$

The expression corresponding to (2-10) for small $\phi$ is [7, pp. 234 and 484, and 19, p. 64].

$$
\begin{gathered}
\sum_{n=1}^{\infty}\left(1 / n^{2}\right) \exp \left(-n^{2} \phi\right) \\
\approx+\left(\pi^{2} / 6\right)-\sqrt{\pi} \phi^{1 / 2}+(1 / 2) \phi \\
-(\phi / \pi)^{3 / 2} \sum_{n=1}^{{ }^{m i n}{ }^{-1}}\left(1 / n^{2}\right)\left[1-(3 / 2)\left(\phi / n^{2} \pi^{2}\right)+(15 / 4)\left(\phi / n^{2} \pi^{2}\right)^{2}+\ldots\right] \exp \left(-n^{2} \pi^{2} / \phi\right) .
\end{gathered}
$$

The repeated occurrence of powers of $\phi^{1 / 2}$ in these relations is particularly worthy of note. 


\section{Evaluation of $F_{r}(\phi)$ for $r \geqslant 2$}

From $(2-10)$

$$
\begin{gathered}
\operatorname{Lim}_{\phi \rightarrow 0} \sum_{n=1}^{\infty}\left(1 / \beta_{n}^{2}\right) \int_{0}^{\phi} e_{n}^{-\beta_{n}^{2 z}} d z \\
=\operatorname{Lim}_{\phi \rightarrow 0}\left[+(1 / 4) \phi-(2 / 3)(1 / \sqrt{\pi}) \phi^{3 / 2}+(1 / 8) \phi^{2}+(1 / 30)(1 / \sqrt{\pi}) \phi^{5 / 2}+(1 / 96) \phi^{3}+\ldots .\right] \\
=\operatorname{Lim}_{\phi \rightarrow 0} \sum_{n=1}^{\infty}\left(1 / \beta_{n}^{4}\right)\left(1-e^{-\beta_{n}^{2} \phi}\right) .
\end{gathered}
$$

From the relation $[1$, p. 581 , eq (1) with $\nu=0]$

$$
\sum_{n=1}^{\infty} \frac{J_{0}\left(\beta_{n} z\right)}{\beta_{n} J_{1}\left(\beta_{n}\right)}=1 / 2
$$

by multiplying both sides of this equation by $z$, and integrating with respect to $z$ from zero to $x$, the expression

$$
\sum_{n=1}^{\infty} \frac{J_{1}\left(\beta_{n} x\right)}{\beta_{n}^{2} J_{1}\left(\beta_{n}\right)}=x / 4
$$

is obtained. From this

$$
\sum_{n=1}^{\infty} \frac{1}{\beta_{n}^{2} J_{1}\left(\beta_{n}\right)} \int_{0}^{x} J_{1}\left(\beta_{n} z\right) d z=(1 / 4) \int_{0}^{x} z d z
$$

and carrying through the indicated integration

$$
\sum_{n=1}^{\infty} \frac{J_{0}\left(\beta_{n} x\right)}{\beta_{n}^{3} J_{1}\left(\beta_{n}\right)}=(1 / 8)\left(1-x^{2}\right)
$$

From this at $x=0$

$$
G_{2}(0)=\sum_{n=1}^{\infty} \frac{1}{\beta_{n}^{3} J_{1}\left(\beta_{n}\right)}=1 / 8
$$

Multiplying (3-2) through by $x$ and integrating from zero to $z$, it follows that

$$
\sum_{n=1}^{\infty} \frac{J_{1}\left(\beta_{n} z\right)}{\beta_{n}^{4} J_{1}\left(\beta_{n}\right)}=(z / 16)\left[1-\left(z^{2} / 2\right)\right]
$$

At $z=1$, then

$$
\sigma_{0}^{(2)} \equiv \sum_{n=1}^{\infty}\left(1 / \beta_{n}\right)^{4}=F_{2}(0)=1 / 32 .
$$

Combining this result with (3-1), it follows that for small $\phi$

$$
\begin{gathered}
F_{2}(\phi) \\
\approx+(1 / 32)-(1 / 4) \phi+(2 / 3)(1 / \sqrt{\pi}) \phi^{3 / 2}-(1 / 8) \phi^{2}-(1 / 30)(1 / \sqrt{\pi}) \phi^{5 / 2} \\
-(1 / 96) \phi^{3}+\ldots
\end{gathered}
$$

Further examination of this method of successive integrations will show that it can lead to the evaluation of all sums of the form $F_{r}(0)$ and $G_{r}(0)$, and 


$$
\operatorname{Lim}_{\phi \rightarrow 0} \sum_{n=1}^{\infty}\left(1 / \beta_{n}^{2 r}\right) \exp \left(-\beta_{n}^{2} \phi\right)=\operatorname{Lim}_{\phi \rightarrow 0} F_{r}(\phi)
$$

In particular, application of the technique outlined yields for small $\phi$ values

$$
\begin{gathered}
F_{3}(\phi) \\
\approx+(1 / 192)-(1 / 32) \phi+(1 / 8) \phi^{2}-(4 / 15)(1 / \sqrt{\pi}) \phi^{5 / 2}+(1 / 24) \phi^{3} \\
+(1 / 105)(1 / \sqrt{\pi}) \phi^{7 / 2}+(1 / 384) \phi^{4}+\ldots
\end{gathered}
$$

These results do not appear to be well known except for $F_{r}(0)=\sigma_{0}^{(r)}$ which can be found in general by a procedure due to Lord Rayleigh [9]. These quantities are all rational fractions, the first few of which are as follows:

$\begin{array}{cc}r & F_{r}(0)=\sigma_{0}{ }^{(r)} \\ 1 & 1 / 4 \\ 2 & 1 / 32 \\ 3 & 1 / 192 \\ 4 & 11 / 12,288 \\ 5 & 19 / 122,880\end{array}$

For integer values of $r, \sigma_{0}^{(r)}$ is half the coefficient of $z^{2 r-1}$ in the expansion of $J_{1}(z) / J_{0}(z)$. In fact, this follows by integrating $J_{1}(z) /\left[z^{2 r} J_{0}(z)\right]$ around the contour given in section 2 above. ${ }^{3}$ See also, the paper by Speigel [10].

\section{Evaluation of $G_{1}(\phi)$ and $G_{r}(\phi)$}

The temperature $v(R, t)$ in an infinite circular cylinder of radius $a$ and thermal diffusivity $\kappa$, as a function of time, $t$, for the boundary conditions

$$
v(R, 0)=0 \quad v(a, t)=1
$$

is given by [7, p. 328]

$$
v(R, t)=1-2 \sum_{n=1}^{\infty} \frac{J_{0}\left(\beta_{n} R / a\right)}{J_{1}\left(\beta_{n}\right)} \exp \left[-\kappa\left(\beta_{n} / a\right)^{2} t\right]
$$

The function has the Laplace transform

$$
L_{p}[v(R, t)]=+p^{-1} \frac{I_{0}(R \sqrt{p / \kappa})}{I_{0}(a \sqrt{p / \kappa})}
$$

Using the asymptotic expansion for $I_{0}(z)$, eq (2-6) above, it readily follows that

$$
\begin{gathered}
\operatorname{Lim}_{t \rightarrow 0} L_{p}[v(0, t)] \\
=\frac{(2 \pi a)^{1 / 2}}{\kappa^{1 / 4}} p^{-3 / 4}\left[1-(1 / 8)(\sqrt{\kappa} / a) p^{-1 / 2}-(7 / 128)(\sqrt{\kappa} / a)^{2} p^{-1}+\ldots .\right] \exp \left(-a p^{1 / 2} / \sqrt{\kappa}\right)
\end{gathered}
$$

Each of the terms in (4-3) is of the form

$$
p^{-(2 k+1) / 4} \exp \left(-a p^{1 / 2} / \sqrt{\kappa}\right)
$$

${ }^{3}$ This technique for finding $\sigma_{0}^{(r)}$ was suggested by F. W. J. Olver of the NBS, in a letter dated March 29, 1962. 
where $k=1,2,3, \ldots$. . The inverse Laplace transform of this expression is

$$
2^{-1 / 4+(1 / 2) k} \pi^{-1 / 2} t^{-3 / 4+}{ }^{(1 / 2) k} D_{(1 / 2)-k}\left(a^{2} / 2 \kappa t\right)^{1 / 2} \exp \left(-a^{2} / 8 \kappa t\right)
$$

where the $D_{\nu}(z)$ 's are parabolic cylinder functions $[11,12]$. Inserting this expression and

$$
\phi=\kappa t / a^{2}
$$

into (4-3), it follows that for small $\phi$ values

$$
\begin{gathered}
\operatorname{Lim}_{\phi \rightarrow 0} v(0, \phi) \\
\approx(8 / \phi)^{1 / 4}\left[+\quad D_{-1 / 2}(1 / \sqrt{2 \phi})\right. \\
-(1 / 4 \sqrt{2}) \phi^{1 / 2} D_{-3 / 2}(1 / \sqrt{2 \phi}) \\
-\left(7 / 64 \quad \phi \quad D_{-5 / 2}(1 / \sqrt{2 \phi})+\ldots\right] \exp (-1 / 8 \phi) .
\end{gathered}
$$

Carsten and McKerrow [13] have obtained the leading term in (4-7) in a somewhat different form. It is known that $\left[11\right.$, p. 119] ${ }^{4}$

$$
D_{-1 / 2}(x)=(x / 2 \pi)^{1 / 2} K_{1 / 4}\left(x^{2} / 4\right)
$$

where $K_{\nu}(x)$ is the Bessel function of the second kind with a purely imaginary argument [1, p. 77]. From this, then, for small $\phi$

$$
v(0, \phi) \approx\left(1 / \sqrt{\pi \phi)} e^{-1 / 8 \phi} K_{1 / 4}(1 / 8 \phi)[1+\ldots . .]\right.
$$

For some purposes this may be an easier form to use than (4-7)-the $K_{1 / 4}(z)$ values are tabulated [13] for $0<z(0.1)<5.0$ and also for $z=6,8$, and 10 .

Higher terms in $\operatorname{Lim}_{\phi \rightarrow 0} v(0, \phi)$ are of interest and to obtain these, use is made of the asymptotic relation [14, p. 92] as $z \rightarrow \infty$

$$
\begin{gathered}
D_{\nu}(z) \\
\approx\left(z^{\nu} e^{-z^{2} / 4}\right)\left[1-\nu(\nu-1)\left(1 / 2 z^{2}\right)+(1 / 2) \nu(\nu-1)(\nu-2)(\nu-3)\left(1 / 2 z^{2}\right)^{2}+\ldots\right]
\end{gathered}
$$

and from this, it follows that for small $\phi$

$$
\begin{gathered}
D_{-k / 2}(1 / \sqrt{2 \phi}) \\
\approx(2 \phi)^{+k / 4} e^{-1 / 8 \phi}\left[1-(1 / 4) k(k+2) \phi+(1 / 32) k(k+2)(k+4)(k+6) \phi^{2}+\ldots\right]
\end{gathered}
$$

and

$$
v(0, \phi) \approx 2 e^{-1 / 4 \phi}\left(1-\phi+4 \phi^{2}+\ldots\right)
$$

Now since $(\mathrm{cf}(4-1))$

$$
\sum_{n=1}^{\infty} \frac{1}{\beta_{n} J_{1}\left(\beta_{n}\right)} e^{-\beta_{n}^{2} \phi}=(1 / 2)[1-v(0, \phi)]
$$

it follows that for small $\phi$

$$
\begin{gathered}
G_{1}(\phi) \\
\approx(1 / 2)-\left(1-\phi+4 \phi^{2}+\ldots\right) \exp (-1 / 4 \phi) .
\end{gathered}
$$

${ }^{4}$ The expression given in [11, p. 119] is ambiguously, if not incorrectly, printed. Equation (4-8) is the proper form, as can be shown by using the relations [12] $D_{-1 / 2}(x)=2^{-1 / 4} e^{-(1 / 4) x^{2}} U\left(1 / 4 ; 1 / 2 ; x^{2} / 2\right)$ and $[12$, p. 13 , eq 1.8 .7$] U\left(1 / 4 ; 1 / 2 ; x^{2} / 2\right)=\left(x^{2} / 2 \pi^{2}\right)^{1 / 4} e^{+(1 / 4) x^{2}} K_{ \pm 1 / 4}\left(x^{2} / 4\right)$. 
Since

$$
\int_{0}^{\phi} G_{1}(z) d z=\sum_{n=1}^{\infty} \frac{1-\exp \left(-\beta_{n}^{2} \phi\right)}{\beta_{n}^{3} J_{1}\left(\beta_{n}\right)}
$$

integrating (4-13) and using (3-3),

$$
\begin{aligned}
& \begin{aligned}
\operatorname{Lim}_{\phi \rightarrow 0} \sum_{n=1}^{\infty} \frac{1}{\beta_{n}^{3} J_{1}\left(\beta_{n}\right)} \exp \left(-\beta_{n}^{2} \phi\right)= & \operatorname{Lim}_{\phi \rightarrow 0}[+(1 / 8)-(1 / 2) \phi \\
& \left.+(1 / 6) \phi\left(7-4 \phi+8 \phi^{2}+\ldots\right) \exp (-1 / 4 \phi)+(7 / 24) \operatorname{Ei}(-1 / 4 \phi)\right]
\end{aligned} \\
& \text { where }
\end{aligned}
$$

$$
E i(-x) \equiv-\int_{x}^{\infty}\left(z^{-1} e^{-z}\right) d z
$$

is the exponential integral function, described and tabulated in Jahnke-Emde [8]. Asymptotically, for large values of the independent variable

$$
E i(-x) \approx-x^{-1} e^{-x}\left[1-(1 / x)+2(1 / x)^{2}-6(1 / x)^{3}+\ldots\right]
$$

and introducing this in (4-14), for small $\phi$ 's

$$
\begin{gathered}
G_{2}(\phi) \\
\approx+(1 / 8)-(1 / 2) \phi+4 \phi^{2}(1-9 \phi+\ldots) \exp (-1 / 4 \phi) \\
\hline
\end{gathered}
$$

Successive integration in the same way, will yield $G_{r}(\phi)$ for any $r \geqslant 3$. Thus, for example,

$$
\begin{gathered}
G_{3}(\phi) \\
\left.\approx+(1 / 48)-(1 / 8) \phi+(1 / 4) \phi^{2}+36 \phi^{3} \exp (-1 / 4 \phi)+\ldots\right)
\end{gathered}
$$

Differentiation of (4-13) with respect to $\phi$ leads to

$$
\begin{gathered}
G_{0}(\phi) \\
\approx+(1 / 4 \phi)^{2}(1-\phi+\ldots) \exp (-1 / 4 \phi)
\end{gathered}
$$

and further differentiation gives expressions for $G_{-r}(\phi)$ in the limit of small $\phi$.

\section{Computation of $\sigma_{0}^{(3 / 2)}$ and $\sigma_{0}^{(5 / 2)}$}

A general method for finding

$$
\sigma_{0}^{(r)}=F_{r}(0)=\sum_{n=1}^{\infty}\left(1 / \beta_{n}\right)^{2 r}
$$

where $r$ is an integer $\geqslant 1$ has been developed above. In the next section, it is necessary to use $\sigma_{0}{ }^{(3 / 2)}$ and $\sigma_{0}{ }^{(5 / 2)}$-i.e., odd rather than even powers of $\beta_{n}$ occur in the sums. In contrast to the previously considered case, it does not appear possible to express the sum of the odd powers of $\beta_{n}$ in closed form. ${ }^{5}$ The numerical computation of $\sigma_{o}{ }^{(r / 2)}$ is simplified by noting that $(1-2)$ yields very good approximations to $\beta_{n}{ }^{-r}$. Thus, for example, the difference between $\left(1 / \beta_{n}\right)^{3}$ and $\left(1 / \beta_{n}{ }^{*}\right)^{3}$ is very small when only the first three terms of the asymptotic series are taken, e.g.,

\footnotetext{
${ }^{5}$ Note the similarity to the sum $\sum_{n=1}^{\infty} 1 / n^{r}=\pi^{r} / \nu(r)$, where $\nu(r)$ is an integer for even values of $r$; but the sum cannot be reduced to a closed form for odd $r$ [6, p. 140].
} 


\begin{tabular}{|c|c|c|}
\hline$n$ & $\left(1 / \beta_{n}\right)^{3}$ & $\left(1 / \beta_{n}{ }^{*}\right)^{3}$ \\
\hline 1 & 0.0719033694 & 0.0717248723 \\
\hline 2 & .0059451708 & .0059451433 \\
\hline 3 & .0015430866 & .0015430863 \\
\hline
\end{tabular}

This suggests writing [15]

$$
\sigma_{0}^{(r / 2)} \equiv \sum_{n=1}^{\infty}\left(1 / \beta_{n}\right)^{r}=\sum_{n=1}^{\infty}\left[\left(1 / \beta_{n}\right)^{r}-\left(1 / \beta_{n}\right)^{r}\right]+\sum_{n=1}^{\infty}\left(1 / \beta_{n}\right)^{r} .
$$

Using the asymptotic relation (1-2), terminated at the $\alpha_{3}$ term, for $\beta_{n}{ }^{*}$, the sums can be evaluated in terms of tabulated functions - the polygamma function [16, p. 9], the sums of negative powers of the integers [16, pp. 244, 304], and Euler numbers [16, p. 276, etc.]. Combining these relations, it is found that

$$
\sigma_{0}^{(3 / 2)}=0.08088147
$$

with an uncertainty of no more than one in the last place given. Similarly, using the same techniques, it has been found that

$$
\sigma_{0}^{(5 / 2)}=0.01265566
$$

\section{The Sums $M_{r}(\phi)$ and $N_{r}(\phi)$}

The functions $F_{r}(\phi)$ and $G_{r}(\phi)$ are closely related to two other functions

$$
\begin{aligned}
& M_{r}(\phi) \equiv \sum_{n=1}^{\infty}\left(1 / \beta_{n}\right)^{2 r-1} \operatorname{erfc}\left(\beta_{n} \sqrt{\phi}\right) \\
& N_{r}(\phi) \equiv \sum_{n=1}^{\infty} \frac{\operatorname{erfc}\left(\beta_{n} \sqrt{\phi}\right)}{\beta_{n}{ }^{2 r} J_{1}\left(\beta_{n}\right)}
\end{aligned}
$$

which also occur in some cylindrical geometry problems. Here, the complementary error function [17]

$$
\operatorname{erfc}(z) \equiv(2 / \sqrt{\pi}) \int_{z}^{\infty} \exp \left(-\xi^{2}\right) d \xi
$$

From eq (2-10) for small $\phi$

$$
\begin{gathered}
\sum_{n=1}^{\infty}\left(1 / \beta_{n}{ }^{2}\right) \int_{0}^{\phi^{1 / 2}} \exp \left(-\beta_{n}{ }^{2} z^{2}\right) d z \\
\approx \int_{0}^{\phi^{1 / 2}}\left[+(1 / 4)-(1 / \sqrt{\pi}) z+\frac{1}{4} z+\frac{1}{12 \sqrt{\pi}} z^{3}+\frac{1}{32} z^{4}+\ldots\right] d z .
\end{gathered}
$$

By the change of variable $\eta=\beta_{n} z$, the expression on the left of this equation can be evaluated, and it is found that for small $\phi$ values 


$$
\begin{gathered}
M_{2}(\phi) \\
\approx \sigma_{0}^{(3 / 2)}-(1 / 2)(1 / \sqrt{\pi}) \phi^{1 / 2}+(1 / \pi) \phi-(1 / 6)(1 / \sqrt{\pi}) \phi^{3 / 2} \\
-(1 / 24)(1 / \pi) \phi^{2}-(1 / 80)(1 / \sqrt{\pi}) \phi^{5 / 2}+\ldots \ldots
\end{gathered}
$$

So that $M_{2}(0)=\sigma_{0}^{(3 / 2)}$.

Similarly, using (3-6) for small $\phi$

$$
\begin{gathered}
M_{3}(\phi) \\
\approx \sigma_{0}^{(5 / 2)}-(1 / 16)(1 / \sqrt{\pi}) \phi^{1 / 2}+(1 / 6)(1 / \sqrt{\pi}) \phi^{3 / 2} \\
-(1 / 3)(1 / \pi) \phi^{2}+(1 / 20)(1 / \sqrt{\pi}) \phi^{5 / 2}+(1 / 90)(1 / \pi) \phi^{3} \\
+(1 / 336)(1 / \sqrt{\pi}) \phi^{7 / 2}+\ldots
\end{gathered}
$$

It is clear that this procedure can be used to obtain $M_{r}(\phi)$ from the value of $F_{r-1}(\phi)$ in each case. Note that, using the asymptotic series [18, p. 129] for erfc $\left(\beta_{n} \sqrt{\phi}\right)$ for large $\phi$ values

$$
\begin{gathered}
M_{r}(\phi) \\
\approx \frac{1}{\sqrt{\pi}} \phi^{-1 / 2} \sum_{n=1}^{\infty}\left(1 / \beta_{n}\right)^{2 r}\left[1-(1 / 2)\left(\beta_{n}^{2} \phi\right)^{-1}+(3 / 4)\left(\beta_{n}^{2} \phi\right)^{-2}\right. \\
\left.-(15 / 8)\left(\beta_{n}^{2} \phi\right)^{-3}+\ldots\right] \exp \left(-\beta_{n}^{2} \phi\right) \\
=\frac{1}{\sqrt{\pi}} \phi^{-1 / 2}\left[F_{r}(\phi)-(1 / 2 \phi) F_{r+1}(\phi)+\left(3 / 4 \phi^{2}\right) F_{r+2}(\phi)\right. \\
\left.-\left(15 / 8 \phi^{3}\right) F_{r+3}(\phi)+\ldots\right]
\end{gathered}
$$

The $N_{r}(\phi)$ functions are obtained in a similar way, by integration, of the corresponding $G_{r}(\phi)$. By this procedure, it is found that for small $\phi$

$$
\begin{gathered}
N_{1}(\phi) \\
\approx N_{1}(0)-\frac{1}{\sqrt{\pi}} \phi^{1 / 2}+\frac{4}{\sqrt{\pi}} \phi^{3 / 2}\left(1-7 \phi+74 \phi^{2}+\ldots\right) \exp (-1 / 4 \phi)
\end{gathered}
$$


The leading terms in these expressions can be computed by rapidly convergent series to be

$$
N_{1}(0)=0.26659 \ldots . \quad N_{1}(0)=0.054972 \ldots
$$

For large $\phi$ values

$$
\begin{gathered}
N_{r}(\phi) \\
\approx \frac{1}{\sqrt{\pi}} \phi^{-1 / 2} \sum_{n=1}^{\infty}\left[1-(1 / 2)\left(\beta_{n}^{2} \phi\right)^{-1}+(3 / 4)\left(\beta_{n}^{2} \phi\right)^{-2}-(15 / 8)\left(\beta_{n}^{2} \phi\right)^{-3}+\ldots\right] \\
\times \frac{\exp \left(-\beta_{n}^{2} \phi\right)}{\beta_{n}^{2 r+1} J_{1}\left(\beta_{n}\right)} \\
=\frac{1}{\sqrt{\pi}} \phi^{-1 / 2}\left[G_{r+1}(\phi)-(1 / 2 \phi) G_{r+2}(\phi)+\left(3 / 4 \phi^{2}\right) G_{r+3}(\phi)-\left(15 / 8 \phi^{3}\right) G_{r+4}(\phi)+\ldots\right]
\end{gathered}
$$

The assistance of F. W. J. Olver and of John H. Hubbell of NBS is gratefully acknowledged. The manuscript was prepared by L. M. Nicholson whose painstaking care with the difficult mathematical typing is deeply appreciated.

\section{References}

[1] Watson, G. N., Theory of Bessel Functions, 2d ed. (University Press, Cambridge, 1949).

[2] Scarborough, J. B., Numerical Mathematical Analysis (Johns Hopkins Press, Baltimore, 1950).

[3] Churchill, R. V., Complex Variables and Applications 2d ed. (McGraw-Hill Book Co., New York, N.Y., 1960).

[4] Erdélyi, A., Ed., Tables of Integral Transforms, Volume I. (McGraw-Hill Book Co., New York, N.Y., 1954).

[5] Whittaker, E. T., and Watson, G. N., Modern Analysis, 4th ed. (University Press, Cambridge, 1935).

[6] Adams, E. P., Ed., Smithsonian Mathematical Formulae (Smithsonian Institution, Washington, 1957), 3d Reprint.

[7] Carslaw, H. S., and Jaeger, J. C., Conduction of Heat in Solids, 2d Ed. (Clarendon Press, Oxford, 1959).

[8] Jahnke, E., and Emde, F., Tables of Higher Functions, 6th ed., Friedrich Lösch, Ed. (McGraw-Hill Book Co., New York, N.Y., 1960).

[9] Rayleigh, Lord (J. W. Strutt), Proc. London Math. Soc. 5, 119 (1874); Scientific Papers I, p. 190 ; cf also [1] p. 502.

[10] Speigel, M. R., J. Appl. Phys. 24, 1103 (1953).

[11] Erdélyi, A., Ed., Higher Transcendental Functions, Volume II (McGraw-Hill Book Co., New York, N.Y., 1953).

[12] Slater, Lucy J., Confluent Hypergeometric Functions (University Press, Cambridge, 1960).

[13] Carsten, H. R. F., and McKerrow, N. W., Phil. Mag. (7) 35, 812 (1944).

[14] Magnus, W., and Oberhettinger, F., Special Functions of Mathematical Physics (Chelsea, New York, 1949).

[15] Epstein, L. F., and French, N. E., Amer. Math. Monthly 63, 698 (1956).

[16] Davis, H. T., Tables of the Higher Mathematical Functions, Volume II (Principia Press, Bloomington, Ind., 1935).

[17] Peirce, B. O., A Short Table of Integrals, 4th Ed., revised by R. M. Foster (Ginn, New York, 1957).

[18] Dwight, H. B., Tables of Integrals and Other Mathematical Data, 3d ed. (Macmillan New York, 1957).

[19] Titchmarsh, E. C., Introduction to the Theory of Fourier Integrals, 2d ed. (Clarendon Press, Oxford, 1948).

(Paper 68B1-111) 